\title{
Cuando digo intersex. Un diálogo introductorio a la intersexualidad ${ }^{*}$
}

\author{
Mauro Cabral y Gabriel Benzur**
}

Gabriel Benzur: A lo largo de los últimos años, un número cada vez mayor de historiadores/as, antropólogos/as, bioeticistas y teóricos/as del género y la sexualidad han tematizado progresivamente la intersexualidad. Sin embargo, para la mayor parte de quienes no están interiorizados/as en el tema, la cuestión intersex sigue siendo un misterio. ¿Qué es, qué debemos entender cuando alguien habla de intersexualidad?

Mauro Cabral: Uno de los problemas más complejos que enfrentamos quienes trabajamos teórica o políticamente sobre intersexualidad es su inmediata asociación, en nuestro imaginario cultural común, con el hermafroditismo - y de este, a su vez, con un individuo con "ambos" sexos, es decir, literalmente, con pene y vagina (un individuo por lo demás inexistente fuera de la mitología y el arte, a excepción, por supuesto, de quienes se consideran hermafroditas de diseño). ${ }^{1}$ El concepto clave para comprender de qué hablamos cuando hablamos de intersexualidad es el de variación. Por lo tanto, cuando decimos

\footnotetext{
* Esta entrevista fue realizada y editada por Gabriel Benzur y Mauro Cabral en Córdoba, Argentina, entre enero y febrero del año 2005.

** Mauro Cabral, de la Facultad de Filosofia y Humanidades de la Universidad Nacional de Córdoba, Cordoba, Argentina, es miembro del International Board de CLAGS (Center for Lesbian and Gays Studies, CUNY) y coordinador del Area Trans e Intersex del Programa para Latinoamerica y el Caribe de IGLHRC (the International Gay and Lesbian Human Rights Commission); Gabriel Benzur es periodista y activista de derechos humanos, en la actualidad reside en Tel Aviv.

1 Es decir, quienes modifican voluntariamente su morfología corporal a fin de dotarla de rasgos hermafroditas.
} 
intersexualidad nos referimos a todas aquellas situaciones en las que el cuerpo sexuado de un individuo varía respecto al standard de corporalidad femenina o masculina culturalmente vigente. ¿De qué tipo de variaciones hablamos? Sin ánimo de exhaustividad, a aquellas que involucran mosaicos cromosómicos (XXY, XX0), configuraciones y localizaciones particulares de las gónadas - (la coexistencia de tejido testicular y ovárico, testículos no descendidos) como de los genitales (por ejemplo, cuando el tamaño del pene es "demasiado" pequeño y cuando el clítoris es "demasiado" grande de acuerdo a ese mismo standard del que antes hablaba, cuando el final de la uretra está desplazado de la punta del pene a uno de sus costados o a la base del mismo, o cuando la vagina está ausente...). Por lo tanto, cuando hablamos de intersexualidad no nos referimos a un cuerpo en particular, sino a un conjunto muy amplio de corporalidades posibles, cuya variación respecto de la masculinidad y la femineidad corporalmente "típicas" viene dada por un modo cultural, biomédicamente específico, de mirar y medir los cuerpos humanos. $^{2}$

GB: Sin embargo, términos como hermafrodita verdadero y pseudohermafrodita siguen vigentes, no solamente en el imaginario colectivo, sino también como diagnósticos clínicos.

MC: En este momento coexisten dos vocabularios, que remiten a horizontes de inteligibilidad diferenciados - lo cual complica ciertamente la comprensión del repertorio de cuestiones involucradas. Si nos ubicáramos históricamente en la Europa de mediados del siglo XIX, encontraríamos una definición de hermafroditismo muy parecida a aquella que consagra la mitología - es decir, la coexistencia, en un mismo individuo, de rasgos corporales definidos de uno y otro sexo (por ejemplo, de pene y

2 Para una reconstrucción crítica de ese modo de mirar, medir y cortar, véase KESSLER, Suzanne. Lessons from the Intersexed. Durham, Duke University Press, 1998. 
ovários). Much*s hermafroditas famosos de la época, como Herculine Barbin - sobre la que escribiera Foucault - fueron considerad*s hermafroditas en ese sentido. ${ }^{3}$ Hacia finales del siglo $\mathrm{XIX}$, sin embargo, esta laxitud clasificatoria fue reemplazada por el criterio taxonómico que sostiene, hasta nuestros días, la vigencia de la familia conceptual de los hermafroditismos - verdadero, y pseudohermafroditismos masculino y femenino. En 1876, el investigador biomédico T.A.E. Klebs propuso un nuevo sistema clasificatorio, basado en el fuerte desplazamiento que había experimentado la comprensión biomédica de la vida hacia lo que podríamos llamar la constelación endocrina - es decir, a la consagración de las hormonas como principio rector del cuerpo (lo que incluía también, por supuesto, los rasgos sexuales, el deseo, e incluso la "personalidad"). De acuerdo a esta nueva taxonomía propuesta, hermafroditas serían solamente aquellos individuos en los que el tejido ovárico y el tejido testicular se presentaran al mismo tiempo, sin importar la configuración externa de su cuerpo sexuado.

Sin embargo, y tal como Dreger nos advierte, debemos tener cuidado a la hora de explicar el cambio por una única causa. El modelo gonádico de la identidad sexual no se sostenía solamente en este espíritu de época prevalenciente en las ciencias de la vida, sino que también presentaba una ventaja inequívoca respecto de su predecesor: la organización de los individuos de acuerdo a sus pares gonadales permitía identificar inequívocamente un sexo verdadero por cuerpo, en un período de intensa ansiedad social, donde la clarificación y la organización de lo viviente aparecía como un imperativo sociopolítico ineludible. Es el momento en el que el fantasma de la inversión recorría Europa, encarnado en aquello que Vernon Rosario llama la irresistible ascensión de los perversos, la indetenible multiplicación,

\footnotetext{
3 Véase también la interesante reconstrucción de la trayectoria foucaultiana en torno a Herculine Barbin en CAPURRO, Raquel. Del sexo y su sombra. Del misterioso hermafrodita de Michel Foucault. DF, Epele, 2004.
} 
y creciente visibilidad pública de mujeres masculinas y hombres femeninos, unidos por prácticas sexuales aberrantes que comenzaban a configurar una naturaleza humana diferenciada especies, en los términos de Foucault. ${ }^{4}$ En este contexto, las clasificaciones precedentes abrían la posibilidad de que el hermafroditismo multifacético que había prevalecido a lo largo de la historia se convirtiera, científica y políticamente, en el fundamento y la justificación de la inversión, que reingresaría a la moral, podríamos decir, bajo los ropajes de la ciencia. ¿Qué hizo Klebs? Situar el sexo verdadero de cada individuo en el interior invisible de su cuerpo, donde la presencia de ovarios y testículos establecía, más allá de cualquier variación morfológica de los genitales, su identidad sexual verdadera. La constatación del hermafroditismo verdadero, dadas las factibilidades biotecnológicas disponibles, podía solamente ser establecida postmortem, cuando el cuerpo pudiera ser abierto y examinado.

A pesar de la persistencia de la clasificación propuesta por Klebs hasta nuestros días, este modelo clasificatorio tuvo un éxito muy breve en el terreno de la identificación de individuos con corporalidades "ambiguas", dado que fue puesto en jaque por desarrollos biotecnológicos particulares - tales como el de la anestesiología. Con la posibilidad de practicar biopsias a pacientes con vida, la medicina se transformaba entonces, y verdaderamente de modo involuntario, en portador de una mala nueva: la existencia de hermafroditas entre nosotros y nosotras, la posibilidad de un tercer sexo. En esta emergencia higiénica tuvo lugar un giro decisivo en el modo en el que la biomedicina y el derecho lidiaban con la "ambigüedad" corporal de ciertos individuos: es el momento en el que William Blair Bell, entre otros investigadores, comienza a proponer hacia 1915, centrar la atención en el modo en el que aquellos individuos con cuerpos

4 Dreger, Alice. Hermaphrodites and the Medical Invention of Sex. Cambridge, Harvard University Press, 1998; FouCAULT, Michel. Historia de la Sexualidad. I. La Voluntad de Saber. DF, Siglo XXI, 1986; ROSARIO, Vernon. L'irrésistible ascension du pervers. Entre literature et psychiatrie. París, Epel, 2000. 
Mauro Cabral y Gabriel Benzur

"ambiguos" se identificaban y eran identificados por otros y otras - es decir, a los aspectos psico-sociales del sexo, o lo que hoy llamaríamos el género. Tal y como se planteaba en ese momento histórico, si una mujer solicitaba atención profesional por esterilidad, y la búsqueda médica revelaba la existencia de testículos, su reasignación al sexo masculino tendría el extraño efecto de convertirla, a ella y a su esposo en... homosexuales. Era mucho más lógico respetar tanto el modo en el que esta mujer se identificaba a sí misma y era identificada, situando su identidad sexual en esta narrativa individual y social de sí más que en una supuesta verdad gonádica invisible a los ojos - pero de indudable productividad en términos de caos social, una vez lanzada al mundo por la palabra médica. Hacia 1930, los avances en el campo de la cirugía reconstructiva permitieron la realizaron de las primeras cirugías de "cambio de sexo", con lo que la capacidad de intervenir sobre el cuerpo para modelar una apariencia acorde entre identidad sexual psicosocial y anatomía en casos de "ambigüedad" pasó a constituir una herramienta sociomédica de primer orden. ${ }^{5}$

GB: De allí ya estamos a un paso de la construcción social del género, de un nuevo paradigma de la identidad. ¿Cómo caracterizar este nuevo enfoque?

MC: A mí entender es posible presentar este paradigma identitario como una conjunción de versiones muy particulares de (pseudo) humanismo y (pseudo)constructivismo. Lo que este paradigma viene a cuestionar, en primer lugar, es la existencia de algún determinante específico, bioanatómico, de la identidad sexual no solamente la identidad sexual de aquellos individuos que hoy llamaríamos intersex, sino la de todos los seres humanos. El sexo mismo comenzaba a aparecer bajo las características dispersas,

${ }^{5}$ Hausman, Bernice. Changing Sex. Durham, Duke University Press, 1998; MeYerowitz, Joanne. How Sex Changed. Cambridge, Harvard University Press, 2002. 


\section{Cuando digo intersex}

múltiples, que le reconocemos hoy - como sexo cromosómico, gonádico, genital... ¿Cuál, de todos ellos, determinaba finalmente la identidad de alguien como niña o niño, hombre o mujer? Entre las décadas de 1950 y 1960 un conjunto de investigadores e investigadoras - esta vez en los Estados Unidos - continuaron el sesgo psicosocial inagurado hacia finales del siglo XIX, afirmando la centralidad de lo que, con nuestro vocabulario actual, podríamos llamar el proceso de generización - es decir, el proceso por el cual un individuo sexualmente neutro era introducido en la femineidad y la masculinidad a través de la socialización (en lo que llegó a ser conocido como el sex of rearing, es decir, el sexo de crianza), cualquiera fuera su corporalidad inicial. Para nombrar el resultado de ese proceso, diferenciándolo de los caracteres específicamente bioanatómicos, se introdujo la distinción entre género, como construcción psicosocial y sexo, como bioanatomía. Sin embargo, y a pesar de la apariencia decisivamente constructivista y corporalmente emancipada de este paradigma, la dependencia respecto del cuerpo sexuado y su morfología seguía siendo fortísima.

GB: ¿Por qué?

MC: Porque la socialización (el proceso de generización) precisaba de un cuerpo donde asentarse, de una base material. Para socializar a alguien como una niña, para que su identidad femenina resultara "exitosa" y sin fisuras, era imprescindible que su cuerpo fuera, en su apariencia exterior, el de una niña standard, capaz de sostener la mirada y la palabra, constitutivas, de su madre y su padre, su propia percepción de sí como ser sexuado. El cuerpo regresaba, por lo tanto - no bajo la forma de una determinación a priori, biológica - sino como el sostén material, imprescindible, de la asignación de género y del éxito de esa asignación a lo largo de la vida. Este regreso del cuerpo sexuado como determinante - esta vez no de la identidad sexual "verdadera", sino de la posibilidad misma de una identidad sexual - precisaba no solamente de asegurar la apariencia exterior de los 
genitales sino también ciertas funciones estimadas fundamentales. El género no se anudaba por lo tanto, desde un principio, y como gesto fundante, instituyente, de una subjetividad genérica, legal, lingüísticamente posible, solamente a través de su inscripción literal en el cuerpo, sino que esa literalidad se extendía a la proyección de prácticas constitutivas de la feminidad y la masculinidad - tales como la penetrabilidad de las mujeres, o la capacidad de penetrar y para orinar de pie en los hombres. ${ }^{6}$

De esta descripción del proceso de generización se derivaban, por lo tanto, los protocolos atencionales aún vigentes en nuestras sociedades: a la asignación temprana al género femenino o masculino debe seguir, de modo imprescindible, la intervención "normalizadora" sobre el cuerpo, capaz de situarlo inequívocamente en el standard masculino o femenino. El paradigma identitario del que hablamos incluía una temporalidad específica, antes que la core gender identity (es decir, el sentido inmodificable que cada cual poseería de ser una mujer o un hombre) se estableciera, hacia los dos años de edad. Cualquier reasignación posterior era muy arriesgada, y virtualmente imposible, por lo que en ese entonces, como en este momento, cada nacimiento intersex era tratado como una verdadera emergencia médica - a pesar de que las variaciones corporales asociadas con la intersexualidad rara vez comportan algún riesgo para la salud.

GB: ¿Pero qué ocurría en aquellos casos donde a una asignación inicial proseguía una re-asignación?

MC: Diferentes autobiografías intersex - incluyendo, por supuesto, la de David Reimer, quien fuera "protagonista" del llamado caso John-Joan, y la de Cheryl Chase, fundadora de la ISNA (Intersex Society of North America), entre otr*s - dan testimonio de uno de

${ }^{6}$ FAusto-Sterling, Anne. Sexing the Body. Los Angeles, Basic Books, 2000; ButLER, Judith. Undoing Gender. New York, Routedgle, 2004; KESSLER, S. Lessons from the... Op.cit.; DREGER, A. Hermaphrodites and the... Op.cit. 
los procedimientos sociomédicos más oscuros asociados a la intersexualidad. Puesto que la generización debe producirse sin "fallas", en aquellos casos donde quienes realizaron una primera asignación cambiaron luego de parecer, el mandato fue un cambio drástico - de nombre, de ropa, de juguetes, pero también de vecindario, e incluso de ciudad, el ocultamiento o incluso la destrucción de fotografías, de documentos... Es decir, la transformación de la historia personal vivida hasta el momento en una prehistoria, tan prescindible como peligrosa (sobre todo para ese individuo cuya subjetividad aparecía entonces como dañada desde un principio).

Sin embargo, debemos tener muy presente que no todas las ocurrencias de variaciones asociadas con la intersexualidad implican algún tipo de dificultad en la asignación de género, o un procedimiento de reasignación de género. Pensemos, por ejemplo, en una niña con cromosomas XX, con ambos ovarios, útero, un clítoris de tamaño médicamente "adecuado"... pero sin vagina. O en un niño con testículos en su posición "correcta", con un pene que ha de crecer... pero cuya uretra finaliza en un costado del pene, y no en la punta. En el primer caso, se estimará que esa niña precisa de una vaginoplastia no para ser nombrada como una niña, sino para asegurar su generización a través de la experiencia corporal; en el segundo, se estimará que ese niño precisa de una cirugía correctiva para asegurar su generización masculina. De esta manera, el cuerpo no aparece genéricamente codificado como marcador inagural del género, sino como condición imprescindible para una biografía que se despliega anticipadamente en términos genérico-sexuales heteronormativos.

GB: El movimiento intersex ha criticado repetidamente el sesgo de género de los protocolos de atención a niños y niñas intersex. ¿En qué consiste ese sesgo?

MC: Los protocolos atencionales se encuentran atravesados no solamente por un profundo sesgo de género, misógino, sino también por un violento sesgo homofóbico. En primer término, y 
en este punto estamos en el reino de la "racionalidad" biomédica, es más fácil hacer una mujer que un hombre, puesto que la femineidad es frecuentemente reducida a la combinación de un clítoris que no pueda ser confundido con un pene por su tamaño, y la capacidad de ser penetrada vaginalmente en una relación heterosexual "normal". Por el contrario, la masculinidad es cuidadosamente reservada sólo para aquellos individuos capaces de conformar el estereotipo peneano de nuestra cultura, enviando hacia la femineidad a todos aquellos que fracasan en esta empresa - paradigmáticamente, a quienes nacieron con micropenes, cuyo tamaño les impediría, desde este punto de vista, habitar exitosamente lo masculino. El pene aparece como un órgano irreproducible - no imitable, no ironizable, podríamos decir, no parodiable (y toda imitación sería, en definitiva, una parodia). Por el contrario, la femineidad es un hueco. La "amenaza" que suponen los clítoris virilizados, es decir, aquellos que miden más de cinco centímetros, es conjurada mediante la práctica regular de clitoridectomías.

El funcionamiento de los protocolos atencionales dispuestos para niñ *s intersex mantienen aún en nuestros días la concepción de la homosexualidad como una posibilidad siniestra derivada de una generización fallada de un cuerpo "malformado" o "defectuoso". Sin embargo, no se trata solamente de que un clítoris virilizado o una vagina ausente conduzcan a una identidad sexual equívoca - lésbica o masculina - o que un micropene produzca homosexualidad mediante prácticas feminizantes (como orinar sentado) como a través de imposibilidades constitutivas (penetrar a una mujer). Estos protocolos de atención instituyen también un cierto saber sobre el mismo deseo heterosexual sobre el cual se consideran expertos $-y$ tal vez custodios (puesto que afirman quién será deseado o deseada, por quién y para qué).

GB: ¿Cuál es la justificación moral de las intervenciones?

MC: Existen dos temores principales, muy generalizados. En primer término, el temor a que si no se realiza una intervención (o 
varias intervenciones) para "normalizar" la apariencia de los genitales, ese individuo se quedará "sin género", o "fuera del género". Esta relación entre genitalidad e identidad es perfectamente comprensible, sumidos como estamos en el funcionamiento naturalizado de un paradigma que fija el género como identidad sexual verdadera - em la genitalidad. Sin genitales "congruentes" al género de asignación, daría la impresión de que no hay sujeto, puesto que no habrá generización posible; es más: parecería que no hay cuerpo, todavía, sino carne desorganizada, que no hace cuerpo. En segundo término, el temor a la discriminación a la que se vería expuest* quien anduviera por el mundo marcad* por una diferencia tan fuertemente estigmatizadora. Por lo tanto, intervenir quirúrgicamente aparece como una medida imprescindible - puesto que, en un mismo gesto humanitario, ahuyenta las posibilidades de discriminación y asegura el ingreso de ese individuo en la subjetividad sexuada, en la ley, en la lengua.

Lo cual introduce, desde mi punto de vista, dos cuestiones de abordaje imprescindible. En primer término, confrontar el temor a la diferencia intersex con la evidencia irrefutable de la diferencia creada por las mismas intervenciones - que va desde la insensibilización de tejidos y la esterilidad a la diferencia éticopolítica de quienes sufrimos intervenciones que modificaron nuestro cuerpo de modo irreversible sin tener ni la oportunidad de consentirlas o rehusarlas ni, en muchos casos, la información fehaciente acerca de lo ocurrido. En segundo término, la pregunta por la definición misma de humanidad sexuada que ha informado e informa el discurso y las prácticas de derechos humanos. En este punto, las intervenciones "normalizadoras" aparecen como auténticos procedimientos de humanización - aunque sean denunciadas por el activismo intersex como procedimientos brutales, inhumanos. ¿Podemos ser sujetos de los derechos humanos quienes, corporalmente, desmentimos la corporalidad normativa de los seres humanos? ¿O necesitamos más bien de un poshumanismo, que no se detenga en los límites de la diferencia 
sexual y de los cuerpos? ¿Cómo incluir, por ejemplo, en las agendas de derechos reproductivos centradas en las "mujeres" a quien sólo será llamada mujer tras sufrir la mutilación de su cuerpo? Remitir toda la cuestión intersex a la esfera biomédica es negarnos a enfrentar uno de los modos privilegiados y más invisibles a través de los cuales el género se instituye y se corporiza.

GB: ¿En qué consiste la intersexualidad como subjetividad política?

MC: Es común que la gente suponga que un cuerpo cuyas características varíen respecto del standard masculino o femenino del modo en el que me he referido anteriormente constituya, por sí mismo, una cierta subjetividad intersex. Eso no es cierto, por varias razones. En primer término, debemos recordar que la intersexualidad se inscribe en los cuerpos a través de una operación biopolítica de generización, sin la cual se trata de un cuerpo no marcado como intersex. De esta manera, es posible encontrar a hombres y mujeres, cualquiera sea su orientación o sus prácticas sexuales, se trate de personas transgenéricas o no, cuyos cuerpos pueden variar en mayor o menor medida del standard, pero para quienes la intersexualidad es completamente ajena. En segundo término, existen personas para quienes la intervención biomédica ha creado un status de "normalidad" corporal, para quienes la intersexualidad es una condición que han dejado atrás en sus vidas - del mismo modo que muchos de quienes se identifican en la versión psiquiátrica del transexualismo, y que "desaparecen" como transexuales para emerger, postransicionalmente, como hombres o mujeres. También existen personas que no fueron intervenidas, y que vivieron su diferencia corporal como un terrible castigo, como un padecimiento, y que no reivindican para sí una intersexualidad - ni como subjetividad, ni como identidad política, inscribiéndose más bien como hombres o mujeres a quienes se vedó la posibilidad de una vida feliz. Porque no debemos olvidarnos, ni por un minuto, de que los 
protocolos atencionales nos hablan del mundo, de este mismo mundo en el que vivimos, donde pareciera no haber espacio para nosotr*s.

¿Quiénes nos identificamos, entonces, como intersex? Por lo general, aquellas personas que fuimos diagnosticadas, y que sufrimos la intervención biomédica. En ese sentido, gran parte del activismo intersex no se funda solamente en la experiencia de la diferencia corporal, sino también en la de aquella otra diferencia, que podríamos llamar ética, de la intervención médica.

GB: ¿De qué experiencia se trata?

MC: Distintos escritores intersex - pienso en Hale Hawbecker en este momento - hablan de cuerpos no intervenidos y de la experiencia brutal de la mirada y la palabra de los otros y las otras. Si bien sus padres lo aceptaron como un hombre con un pene muy pequeño, él refiere el momento en el que su cuerpo es mirado y marcado por una expresión médica horrorizada: "¿quién te hizo esto?". ${ }^{7}$ La intersexualidad como sitio es la producción de ese horror, que excede al médico, que habla de un entramado de los cuerpos en la cultura. Sin embargo, la experiencia de much*s activistas intersex, me atrevería a decir, de la mayoría, es la del cuerpo intervenido, lo cual crea una multiplicidad de cuerpos en juego: por un lado, el cuerpo vivido cotidianamente, marcado, cortado y cosido, insensibilizado; por otro lado, el cuerpo perdido, que puede imaginarse o recordarse, fantasearse, el cuerpo que hay que reconstruir, la experiencia de la historia personal robada, de la historia clínica ocultada, falseada o destruida. En ese sentido, la experiencia intersexual es la de un pathos trágico, en su sentido aristotélico, la de un camino de autodescubrimiento que no se resuelve en ninguna redención. La subjetividad intersex se funda en la intensidad de una experiencia del extrañamiento. En ese sentido, la intersexualidad es un producto paradójico, cruelmente

7 HAWBecker, Hale. Who Did This to You. In: Dreger, Alice. (comp.) Intersex in the Age of Ethics. Hagerstown, Md, University Publishing Group, 1999. 
paradójico, de los procedimientos médicos destinados a erradicarla.

GB: ¿Qué demanda el activismo político intersex y cuáles han sido sus logros más importantes hasta ahora?

MC: Nuestras demandas son simples, y comienzan, como afirma Cheryl Chase, con la reubicación del problema - del sistema biomédico, al sistema cultural de los géneros en el que la biomedicina funciona, y la estigmatización de los cuerpos que varían. Si persiste el modelo actual de asignación, binario, se demanda la asignación de cada individuo al género femenino o masculino, de acuerdo a las mejores expectativas de una vida feliz, $y$ teniendo en cuenta, decisivamente, la experiencia de asignaciones anteriores; pero esa asignación inicial no debe implicar la modificación quirúrgica, "cosmética", del cuerpo, dándole a ese niño o niña la posibilidad de decidir acerca de la necesidad de modificarlo o no en el futuro, con pleno conocimiento de las consecuencias que cada decisión comporte. ${ }^{8}$

Hemos logrado que nuestra existencia sea. El trabajo pionero de Cheryl Chase y de la Intersex Society of North America (ISNA) lograron algo fundamental: la transmisión. ¿Qué es lo que repite, como un mantra? Hay una vida posible. No estas solo. No estas sola. No estas sol*. No sos un monstruo. Y si sos un monstruo, bienvenid* seas: no sos el únic*. Hemos podido llorar y consolarnos junt*s. Hemos contado nuestras historias a quienes podían, carnalmente, reconocerlas como suyas. Ha habido también un conjunto importante de intervenciones públicas. El amicus curiae que la ISNA presentó a la Corte Constitucional de Colombia en 1999, por ejemplo, constituyó un elemento central en esos dos fallos históricos que, hasta la actualidad, son el mejor testimonio del modo en el que el sistema jurídico-normativo puede proteger la integridad corporal y la autonomía decisional de

8 CHASE, Cheryl. Mapping the Emergence of Intersex Political Activism. GLQ: A Journal of Gay and Lesbian Studies, vol.4, n² 2, Spring 1998. 
niñ ${ }^{*}$ s intersex. ${ }^{9}$ Pero también le hemos dado a otras familias la oportunidad de encontrarnos, de conversar con nosotr*s aquello que rara vez pueden conversar con profesionales: los resultados vividos. Hemos introducido un nuevo vocabulario, complicando la lengua, agregando asteriscos, arrobas, imposibilidades del decir, le hemos dado un trabalenguas a la lengua, la hemos puesto a tartamudear. ¿Hemos detenido algún cuchillo? Eso es lo que espero.

GB: Pero ¿̇y qué hay de la representación política?

MC: Ese es uno de los aspectos más interesantes - al menos en mi opinión - de la introducción de agendas intersex en movimientos políticos diferentes, tales como el feminismo, el movimiento por los derechos sexuales y los derechos reproductivos, y los movimientos GLTB. ¿Quién hablará, en esos lugares, públicamente, de intersexualidad? Sin lugar a dudas, contamos con aliados y aliadas muy valiosos, aquellas personas que introducen cuestiones intersex allí donde nosotr*s aún no podemos ingresar - paradigmáticamente, en aquellos espacios feministas reservados "solo para mujeres" a los que muchas personas intersex tenemos vedada la entrada. Sin embargo, nuestra tendencia política fundamental es el pedido - cuando no la exigência - de contar con la posibilidad de hablar en primera persona. Lo cual plantea, indudablemente, la adhesión a una cierta política de las identidades. Y es que en realidad se trata de una trampa. Cuando el activismo intersex - al menos aquel en el que mis compañeros y yo nos reconocemos - reclama su derecho a la primera persona enunciativa, ese reclamo no deja de ser una trampa, una tentación, podríamos decir. Puesto que no hay una evaluación acerca de quién podría nombrarse y quién no como intersex, si se de lo que se trata es de asumir, en el acto enunciativo, la intersexualidad como sitio, como "identidad"

9 Estos documentos pueden encontrarse en el sitio web de la ISNA, www.isna.org. 
política: cualquier sujeto, cualquier cuerpo puede habitarla. La única condición, entonces, es el compartir la misma suerte - no hablar de, no hablar por, hablar como, el "yo, intersex" que constituye a alguien, a cualquiera, en sujeto de un desplazamiento fortísimo, tanto del decir como de la escucha.

GB: Si consideramos lo que decías antes, respecto del sesgo de género y la homofobia de los protocolos intersex, la alianza con el movimiento feminista y el movimiento GLTB parece fundamental.

MC: Es cierto, y sin duda lo es. Sin embargo es una alianza que no se da sin dificultades. Creo que el problema central pasa por el modo en que los diferentes sujetos políticos son constituidos, a través de ejes corporales, genéricos, sexuales... Si asumimos que el sujeto del feminismo son las mujeres, y la definición de mujer (y de hombre) no es puesta siquiera en duda, difícilmente la introducción de cuestiones intersex llegue a buen puerto, porque el activismo intersex viene a cuestionar, justamente, el funcionamiento del Género, el carácter prescriptivo de la diferencia sexual, que en muchos casos el feminismo acepta acríticamente y que, en otros casos, eleva a categoría ontoepistemológica - recordemos, por ejemplo, a Luce Irigaray en Speculum. El funcionamiento de ciertos espacios de exclusión por ejemplo, las travestis que no pueden ingresar por carecer de la experiencia de la mestruación - veda la entrada a la mujer intersex que fue mutilada para ser enunciada, justamente, como una mujer. Es extraño, ¿no? O quizás no lo es, si recordamos aquello que decía Judith Butler en Cuerpos que Importan: algunos cuerpos no importan, ciertas materialidades no cuentan, pero no como resto inservible, sino como condición de posibilidad fundamental para lo que sí importa, para lo que sí cuenta - esos otros sujetos de la enunciación, niños y niñas perdidos, que 
existen en el género en virtud de una exclusión fundante, de una abyección primera. ${ }^{10}$

En el caso del movimiento GLTB existen problemas algo diferentes, a nivel de dos lógicas contrapuestas de diversidad, fundadas en políticas muy arraigadas de la identidad sexual. Por un lado $-\mathrm{y}$ hablo prioritariamente de mi experiencia regional - se concibe la diversidad como la coexistencia respetuosa de "identidades" diferentes, organizadas en torno a cuestiones diferentes. Ahora bien, ¿qué hay acerca de nuestra posibilidad de ser gays y lesbianas? ¿Existe la posibilidad de ser gay sin pene, por ejemplo? En ese sentido, introducir agendas intersex exigiría, a mi entender, cuestionar profundamente los supuestos corporales que sostienen esas "identidades" y "orientaciones sexuales".

GB: La escritura autobiográfica, testimonial ¿puede ser considerada el estilo intersex por antonomasia?

MC: El rol de las personas intersex como testimoniantes es sin lugar a dudas fundamental, pero también un arma de doble filo; por un lado, la producción autobiográfica ha sido y es uno de los instrumentos más importantes de intervención intersex en la cultura - no solamente en cuanto a la capacidad crítica que despliegan much*s activistas, sino también al mismo acontecimiento, al que la autobiografía intersex tenga lugar, que el decir intersex tenga lugar, ocurra. Ese decir funciona como una cuña, como una fisura que se va abriendo, muy lentamente, en la escucha reducida. Y ese es uno de los aspectos más sombríos del activismo intersex: nuestra posición como enunciatarios, como pacientes, es decir, sujetos de una tradicional escucha menguada, mutilada. Por lo general no se trata de nuestra expertise - ino somos médicos, no somos médicas! - sino más bien del modo en que somos confinados al sitio de aquel que habla desde el dolory solo desde el dolor -, cuyo discurso lleva impresa la marca de un

${ }^{10}$ Butlen, Judith. Cuerpos que Importan. Sobre los limites discursivos y materiales del sexo. Buenos Aires, Paidós, 2002. 
idiotismo, la ley del lugar, diría Michel de Certeau, cuya experiencia no es generalizable, puesto que se trata, a lo sumo, y en el peor de los casos, de un accidente. Sin embargo, existe otra cuestión, a la que podríamos llamar, mínimamente, la cuestión del vocabulario o, si intentamos una consideración de máxima, la cuestión de la lengua. Una revisión rápida de los textos que incluyen nuestra propia comprensión aparece estructurada, dicha, en el vocabulario que emergió, triunfante, de los años '50 y '60: género, identidad de género, orientación sexual son parte de nuestra comunicación cotidiana... y forman parte, también, del lenguaje de la subversión. ¿Qué hacer con este vocabulario, entonces? No estoy del todo seguro que debamos centrar nuestros esfuerzos en renovarlo, sino más bien en una estrategia combinada. Historizar, insistir en el carácter metafórico de esos mismos términos - sin olvidar, por supuesto, el modo en el que las metáforas hacen mundo, hacen realidad $-\mathrm{y}$ contaminarlo por dentro, hacerlo estallar; proponer, por supuesto, nuevas metáforas, nuevos usos... La intersexualidad también necesita, en los términos de Blumemberg, de una metaforología. Por otro lado, y si nos olvidamos por un momento del vocabulario específico, aún así debemos lidiar con otro problema - los cuerpos de la lengua, del masculino y el femenino del lenguaje que hablamos, y de su dimensión carnal, normativa... ¿cómo lograr, testimoniando, que nuestro cuerpo imposible se diga, hable, pase, por la lengua?

GB: Sin embargo muchas personas intersex, como vos mismo, eligen identidades o bien masculinas o bien femeninas, y no un más allá o un afuera del género, o una ambigüedad de género.

MC: Es cierto. Y a mí me parece fundamental introducir en este punto una demanda ética de riguroso cuño kantiano: las personas somos fines en nosotros mismos, no fines de la teoría. Existe una expectativa de que la intersexualidad promueva, de algún modo, la emergencia de nuevas identidades, o el deshacerse de las antiguas. En algunos casos es cierto, en otro no. Pero si 
transformamos la intersexualidad en un nuevo deber ser - esta vez de sesgo foucaultiano (pienso en el limbo identitario donde Foucault ubicaba a Herculine Barbin), o queer - el proyecto subversivo fracasa desde el principio. Lo que se introduce es un saber - exactamente la clase de saber clasificatorio que busca subvertirse. Es cierto, muchas personas intersex, incluido yo mismo, nos nombramos como hombres o mujeres; sin embargo, es cierto también que la intersexualidad - y estoy considerando el viejo llamado de Sandy Stone a una visibilidad post-transexual, a no desaparecer, a visibilizar las cicatrices, las eróticas - subvierte la cerrazón del género, donde género podría escribirse perfectamente con mayúsculas, Género, como otra forma de la Ley. Que yo, con mi cuerpo, con mi cuerpo imposible, me diga un hombre, no solamente, como podría pensarse, y quizás correctamente, reinstituye en mi enunciación mínima el sistema bipolar de géneros, sino que también lo ironiza, lo vuelve no sapiente, incierto, lo sume en la incertidumbre. Tal vez, nos dicen, entonces sea imposible saber quién es quién. Pienso, como muchos de mis amigos y compañeros de activismo intersex piensan, en la posibilidad de una nominación otra - ni hombre ni mujer. Pero una vez más, mi temor es la reintroducción de la taxonomía, el "por supuesto, viendo su cuerpo se sabe, claramente que no es ni un hombre ni una mujer - $\mathrm{y}$, por lo tanto, como petición de principio, se sabe lo que es el cuerpo de hombres y mujeres". Y prefiero, por lejos, la opción contaminante, la de la incertidumbre, la del no saber.

GB: ¿Por qué alguien se dedicaría al activismo intersex o, más aún, al activismo intersex en primera persona? ¿Por qué alguien se llamaría intersex públicamente? ¿Constituye el activismo intersex una forma de cura, por ejemplo?

MC: Durante años me he hecho a mí mismo las mismas preguntas. Indudablemente, la primera explicación que surge es el deseo de que las cosas cambien; que nuestra historia no le ocurra a otras personas, a otros niños y niñas, a otros y otras adolescentes. 
Damos testimonio como una manera de introducir, siquiera por un momento, como un relámpago, la existencia de ciertos horrores invisibles en la cultura, lo que está al mismo tiempo en su revés y en sus fundamentos. El gênero, tal y como los feminismos lo proponen, no es solo emancipación: el género hiere, el género mata, el Género - que hablamos y que nos habla, el que nos hace sujetos. La diferencia sexual no solo se celebra, también se construye, laboriosamente se construye, con tijeras, con hilos de sutura, con carne; el cuerpo se hace, no se nace un cuerpo, se llega a serlo, dolorosamente, mutiladamente - como afirman Beatriz Preciado y Monique Wittig, a través de una primera cirugía plástica de inscripción, la de la carne en cuerpo. Ponemos el cuerpo ahí, entonces, donde ponemos la palabra, y cruzamos los dedos, esperando que funcione, en algún lugar, para alguien; que alguna persona, alguna vez, recuerde el relámpago que pasó por nuestro decir, el entreabrirse de ese chirriar quirúrgico, e impida algo. Sin embargo, hay ciertos padeceres que el activismo no cura, porque la mutilación médica abre el espacio a una experiencia particular del género y del socius que no se olvida. El lenguaje aparece en sus costuras, lo real se tensiona, el género está poblado de sujetos ausentes, de cuerpos enmudecidos; la observancia corporal y subjetiva de la Regla - del Género como una Regla - lo invade todo; todo habla de lo que se produce, de lo que se está produciendo en algún otro lugar, de lo que se produjo en nuestro cuerpo. Llevamos encima las marcas de que la generización no se produce sin espanto, sin carne masacrada, en una lengua sin espacio, que afixia, donde las fisuras y los orificios deben abrirse a fuerza de poner el cuerpo, y de obligarlo, semióticamente, a que estalle, poner el cuerpo para hacer posible la contaminación, el estallido del Cuerpo. 
Cuando digo intersex

GB: El estilo de activismo intersex que ustedes encaran considera métodos a menudo no convencionales de intervención. ${ }^{11}$

MC: Hay dos instancias principales de intervención donde nuestro activismo podría ser considerado "no convencional", a pesar de que dudo mucho de la "convencionalidad" de cualquier tipo de activismo intersex. Una primera instancia se relaciona con las formas de representación visual de cuerpos intersex. Existe un modo codificado - el estilo del manual médico, podríamos decir en el que nuestros cuerpos aparecen por lo general desnudos, con los ojos o el rostro entero cubierto por un rectángulo o un círculo, negro o blanco, apoyados contra algún tipo de instrumento de medición; o bien la fotografía en primer plano de los genitales de alguien, que permanece oculto como tal frente a la cámara, con un dedo que los abre y los muestra, a veces como forma de comparación - entre el tamaño del clítoris y el del dedo índice que lo señala, por ejemplo. El activismo intersex norteamericano pienso, por ejemplo, en el número de la revista Chrisalys dedicado a la intersexualidad, o en la maravillosa compilación de Alice Domurat Dreger, Intersex in the Age of Ethics - há trabajado intensamente en la proposición de otro estilo representacional: las personas retratadas aparecen vestidas, sonrientes, en sus casas, como personas, con sus mascotas (como la famosa fotografía de Cheryl Chase con su caballo). ${ }^{12}$ Existe un indudable gesto de afirmación, un grito que atraviesa las fotografías y resuena en nuestros oídos: somos humanos. Nuestro trabajo a nivel de la representación visual se enmarca en otros proyectos - tales como el encarado por Loren Cameron y, particularmente, por Del La Grace Volcano. Somos, una vez más, intersexuales desnudos, pero en un desnudo celebratorio, es decir, lo que es, sexuado y a la vista, fuera del código representacional biomédico. Esas

${ }^{11} \mathrm{El}$ "ustedes", en este acaso, se restringe al colectivo "Extranjer*s en la Lengua", integrado por Ariel Rojman, Javier L., Dawson Horwitz y Mauro Cabral.

12 Dreger, A. Intersex in the Age... Op.cit. Chrysalis. The Journal of Transgresive Gender Identities. Vol. 2, n 5, Fall 1997-Winter 1998. 
representaciones incluyen filmaciones, cuerpos en acción, en acción erótica, pornográfica: poner en circulación esos cuerpos imposibles del deseo - después de todo, eso es lo que parece haber movido los bisturíes en primer término, "nadie va a desearte, nadie va a quererte". No hay forma para nosotros de clamar por la belleza de nuestros cuerpos sin que el cuerpo esté presente. Es lo que hay. La cicatriz, eso es lo que hay, la marca. La positividad. En ese sentido, nuestro activismo es un intento persistente de desmentir la representación hegemónica en el imaginario occidental - la de la intersexualidad como una forma de falta o de exceso. Una segunda forma de la intervención, muy relacionada con la anterior, es el trabajo en torno al testimonio, al status epistemológico del testimonio y su productividad. Tal y como dije anteriormente, tanto la intersexualidad como la transexualidad aparecen continuamente codificadas, narrativamente, como discursos verdaderos, las expresiones, testimoniales, verdaderas por testimoniales, de un sí mismo por naturaleza no irónico, no ironizable. No hay humor en la identidad de género - aquel sentido interior de ser una cosa y no la otra, y de haberlo sido desde siempre, aquel sentido del que se sabe, positivamente. No hay performance. $\mathrm{Y}$ nosotros creemos, políticamente, en el testimonio como performance. No en nuestro testimonio como performance, sino en los testimonios en general performance estructuradas de acuerdo a tropos culturales, que las vuelven inteligibles. El tropo de la verdad testimonial es sin duda uno de ellos.

GB: Pero la afirmación del testimonio como performance ¿no compromete la eficacia del activismo intersex?

MC: La efectividad del activismo intersex está de hecho comprometida por una multiplicidad de factores: porque somos ex pacientes que contamos historias pesadillezcas; porque nuestras historias desmienten uno de los sueños iluministas más preciados de nuestra cultura; porque esas mismas historias posicionan a nuestros benefactores en la incómoda posición de carniceros 


\section{Cuando digo intersex}

involuntarios; porque estamos allí para decir "no, no resultó". Y también está comprometida por el ideal de autenticidad que va desde la interioridad generizada de la comprobación pericial de la identidad, tan frecuente en el "cambio de sexo" ( $\mathrm{y}$ en quienes dicen "esperemos el sexo cerebral verdadero para asignar"), por el lugar de la verdad en la cultura y del testimonio como puesta irrefutable de la verdad. Sin embargo, creemos que nuestra propuesta apunta a un cambio que tiene lugar en otro sitio, y es un cambio fundamental: el género como performance, la copia de la copia, la escucha mutua... Las personas que nos escuchan, que prestan atención a nuestras historias, participan de la misma cultura de la que nosotros participamos, cuentan con recursos tropológicos semejantes, intercambiamos los mismos mitos fundantes, en una lengua que nos traiciona todo el tiempo y que nos traiciona por igual. ${ }^{13}$ Nuestra pesadilla no habla solamente de nuestros propios cuerpos, habla de los suyos, del modo en el que llegaron a ser, genéricamente, quienes son. Damos testimonio del trabajo de la Verdad entre nosotros, entre nosotros y ellos, la distancia, Verdadera, que se ha trazado, el trazado de ese límite, contamos historias, y al contarlas, intentamos desesperadamente que lo que se escuche es el propio discurrir del Género como una historia.

${ }^{13}$ Frank, Victor. The Wounded Storyteller: Body, Illness and Ethics. Chicago, Chicago University Press, 1995. DerRIDA, Jacques e Dufourmantelle, Anne. La Hospitalidad. Buenos Aires, Ediciones de la Flor, 2000. 